\title{
Visor de Espacios Urbanos Sensibles (VEUS). Una nueva herramienta para intervenir en la ciudad.
}

\author{
Rafael R. Temes Córdovez \\ Departamento de Urbanismo, Universidad Politécnica de Valencia, Valencia, España \\ E-mail: rtemesc@urb.upv.es
}

\begin{abstract}
Resumen. En el año 2018 la Dirección General de Vivienda, Rehabilitación y Regeneración urbana, de la Comunitat valenciana presentó el "Visor de Espacios Urbanos Sensibles de la Comunitat Valenciana (VEUS)". Este visor, tiene como objetivo, identifica a través de cartografia las "áreas urbanas sensibles", entendiendo como tales, las zonas urbanas donde las dimensiones socio económicas, residenciales y socio demográficas sean notablemente menores que la media de la Comunitat Valenciana. Este trabajo es de carácter similar, tanto por sus contenidos como por sus objetivos, a los desarrollados recientemente por Castilla y León a través de su "Estrategia de Regeneración Urbana en Castilla y León (ERUCYL)"; el desarrollado por el Ayuntamiento de Madrid a través del "Banco de Indicadores para la determinación de la vulnerabilidad integral" así como el Plan Madrid Recupera (MAD-RE) o los trabajos desarrollados hace unos años en Andalucía "Vulnerabilidad del tejido social de los barrios desfavorecidos de Andalucía". En todos ellos, a partir de un análisis estadístico basado en un conjunto de variables consideradas clave para la identificación de situaciones de desfavorecimiento o vulnerabilidad, se determina, a diferentes escalas, la naturaleza de los problemas. A partir de estos antecedentes, el trabajo que se presenta ofrece una propuesta para medir el nivel de vulnerabilidad residencial, socio-económico y socio-demográfico de las secciones censales de la Comunitat Valenciana. Se muestra sus principales resultados y posibles mejoras
\end{abstract}

Palabras clave: Vulnerabilidad, obsolescencia, regeneración urbana.

\section{Antecedentes}

La Dirección General de Vivienda, Rehabilitación y Regeneración urbana, durante el año 2016, en colaboración con el Instituto Cartográfico de Valencia y el Instituto Valenciano de la Edificación, elaboró una primera aproximación al "Visor de Espacios Urbanos Sensibles de la Comunitat Valenciana (VEUS)". Este visor, tiene como objetivo, identifica a través de cartografía las "áreas urbanas sensibles", entendiendo como tales, las zonas urbanas donde las dimensiones socio económicas, residenciales y socio demográficas sean notablemente menores que la media de la Comunitat Valenciana.
Este trabajo emprendido por la Generalitat, es de carácter similar, tanto por sus contenidos como por sus objetivos, a los desarrollados recientemente por Castilla y León a través de su "Estrategia de Regeneración Urbana en Castilla y León (ERUCYL)"; el desarrollado por el Ayuntamiento de Madrid a través del "Banco de Indicadores para la determinación de la vulnerabilidad integral" así como el Plan Madrid Recupera (MAD-RE) o los trabajos desarrollados hace unos años en Andalucía "Vulnerabilidad del tejido social de los barrios desfavorecidos de Andalucía". En todos ellos, a partir de un análisis estadístico basado en un conjunto de variables consideradas clave para la identificación de situaciones 
de desfavorecimiento o vulnerabilidad, se determina, a diferentes escalas, la naturaleza de los problemas. Esta identificación servirá luego para establecer un orden de prioridades a la hora de aplicar las políticas para la mejora de la vivienda y la vida de los ciudadanos.

Estos primeros resultados indican la necesidad de complementar el trabajo con bases de datos e información que permitan caracterizar de forma más precisa las áreas estudiadas, así como contrastar y validar la metodología utilizada para establecer los indicadores sintéticos generados. Para ello, en junio de 2017 la Subdirección General de Vivienda y Regeneración Urbana encarga un "Informe de validación y mejora de la información contenida en el Visor de Espacios Urbanos Sensibles de la Comunidad Valenciana (VEUS)" (Cód. Exp CMENOR/2017/26/31).

\section{Cuestiones previas: definición de vulnerabilidad y áreas urbanas sensibles}

El concepto de vulnerabilidad ha adquirido especial relevancia en las ciencias sociales en los últimos años. Siendo muy utilizado en el caso de la geografía, refiriéndose directamente a las probabilidades de ser afectado negativamente por un fenómeno geográfico y/o climatológico, y también por la economía en relación a la reacción macroeconómico ante shocks externos (French-Davis, 1999), en las ciencias sociales ha tenido un auge especial en la útil década. Según recoge (Rodríguez, 2001) dicho auge parece haberse iniciado por los estudios de Caroline Moser y su grupo en el Banco Mundial, que se sintetizaron en el denominado asset/vulnerability framework (Kaztman y otros, 1999; Mosser, 1998).

Ahora bien, la noción de vulnerabilidad ha superado ampliamente esta delimitación inicial (Rodríguez, 2001). Se ha empleado como un componente de creciente importancia dentro del complejo de desventajas sociales y demográfica que se delinean en la "modernidad tardía" (Rodríguez, 2001). Se ha considerado como el rasgo negativo más relevante del modelo de desarrollo basado en la liberalización económica y la apertura comercial (CEPAL, 2000; Pizarro, 2001). Se ha planteado que es la manifestación más clara de la carencia de poder que experimentan grupos específicos, pero cuantiosos, de la humanidad (Bustamante, 2000). Se ha sostenido que es el reflejo de la gran cantidad de movimientos de entrada y salida a la condición de pobreza (CEPAL, 2000).

Este trabajo utiliza la noción de vulnerabilidad de manera amplia e integral. En palabras de Alguacil (2006), entenderemos la vulnerabilidad como un "término que se refiere a la movilidad social descendente y que viene a significarse como la antesala o caída en la exclusión social y residencial. Con frecuencia se suele referir tanto a colectivos sociales como a territorios o lugares en situación de riesgo o de declive, aunando por tanto el doble vínculo entre espacio y estructura social". De acuerdo con esta conceptualización y acotando el territorio de análisis en lo urbano, debemos definir que entendemos por exclusión o segregación residencial y social, antecedentes de la vulnerabilidad urbana.

Tras el repaso de los términos más utilizados para referirse a estos tipos de áreas (Temes, 2014), podemos concluir en varias ideas:

-La diferencia entre los contenidos de cada una de las denominaciones descritas es prácticamente imperceptible, justificando su adjetivación únicamente por la fuente $u$ origen de donde se derivan.

-En todos los casos la denominación de dichas áreas hace alusión a situaciones provocadas como consecuencia de la combinación de factores físico-urbanos, sociales $y$ económicos, describiéndose la dinámica de su comportamiento a partir de lo que hemos dado en llamar como "la espiral de la obsolescencia", es decir, "el proceso de degradación va aumentando progresivamente a medida que se van incorporando y entrando en sintonía cada uno de los factores anteriores citados"(Temes, 2007, p.53).

-En este trabajo se ha optado por utilizar la denominación integral de Espacios Urbanos Sensibles (EUS) construidas en base a las distintas dimensiones de vulnerabilidad antes expresadas: vulnerabilidad físico-residencial; vulnerabilidad socioeconómica; vulnerabilidad sociodemográfica 


\section{Características del trabajo y naturaleza de las variables}

Las características más destacadas del trabajo son:

-Se trabaja a escala de Sección Censal para toda la CV. Se cuenta con 3.470 recintos de análisis -Se trabaja con las 9 variables que a continuación se relacionan. Para poder trabajar con dichas variables se han efectuado varias peticiones de microdatos confidenciales con finalidad científica al Instituto Nacional de Estadísitica (INE). Dicha petición condiciona, limita y compromete la cesión y el uso de dichas variables exclusivamente para la finalidad declarada en el trabajo del VEUS.

Las condiciones que han guiado la elección del conjunto de variables utilizadas para este trabajo y las fuentes recopiladores de las mismas, se pueden resumir en cuatro criterios básicos:

-Integralidad. Condición que garantiza la valoración de las áreas urbanas desde una perspectiva sociodemográfica, socioeconómica y física-residencial de los espacios urbanos.

-Representatividad. En cuanto a que los datos empleados para este trabajo debían procurar ser los más actuales posibles y abarcar de forma homogénea la totalidad de los ámbitos de análisis. Este punto es muy importante pues ha condicionado, en buena medida la elección de las variables. Al tratase de un trabajo a escala de toda la Comunitat se ha tenido que contar con variables que tuviesen representación en todas las secciones censales para poder hacer una comparación.

-Renovación. En referencia a la vocación, establecida como condición inicial en el trabajo, de revisión temporal de las variables para mantener un seguimiento de la dinámica de las áreas, en función de las medidas adoptadas. Las fuentes de trabajo debían, por tanto, tener la garantía de su continuidad en el tiempo y su extensión al territorio de la Comunitat Valenciana.

-Sistematización. Haciendo alusión a la conveniencia de trabajar a través de un SIG (Sistema de Información Geográfica), que pudiera no solo mantener siempre vinculada la información alfanumérica con la gráfica a través de una estructura sólida, sino que, además, permitiese una fácil actualización de los datos en todo momento.

Para respaldar la elección de las variables finalmente empleadas se han analizado 10 documentos de similar perfil y objetivos, con el propósito de comprobar el tipo de variables usadas, su temporalidad, grado de agregación y metodología de cálculo.

Relación de fuentes consultadas:

-La desigualdad urbana en España. Coordinador: Felix Arias Goytre, 1995

-Áreas vulnerables en el Centro de Madrid. Coordinador: Agustín Hernández Aja, 2005

-Trabajos desarrollados en el CPSV. Identificación, localización y caracterización de las secciones censales desfavorecidas de la Región Metropolitana de Barcelona. Autor: Bernat Goñi Ros, 2007

-El tapiz de Penélope. Transformaciones residenciales sobre tejidos sin valor patrimonial. Autor: R. Temes Cordovez, 2007

-Programa Municipal de Actuaciones para la Periferia de Madrid. Análisis de Polígonos de Bloque Abierto. Coordinador: Juan Manuel Fernández Alonso, 2007

-Vulnerabilidad del tejido social de los barrios desfavorecidos de Andalucía. Coordinadora: Carmen Egea, 2008

-Banco de indicadores para la valoración del suelo urbano consolidado en Madrid. Áreas vulnerables. Dir.: R. Temes Cordovez, 2010 -Plan de Rehabilitación Integral de Castilla y León /Estrategias de regeneración urbana de CyL. Dir. Juan Luis de las Rivas, 2011-2016 -Áreas vulnerables de la ciudad de Valencia. Autor. Oficina de Estadística Ayunt. Vlc, 2016 -Plan Madrid Recupera. Programa MAD-RE. Autor. Ayuntamiento de Madrid, 2017

-La pobreza en España desde una perspectiva de género. Autores: Francesc Valls Fonayet y Ángel Belzunegui Eraso. Fundación Fomento de Estudios Sociales y Sociología Aplicada (FOESSA), 2014.

-Desigualdad, brecha salarial y de género en la Comunitat Valenciana. 2006-2014. Autores: Beatriz Larraz, José Pavía y Encarnación Moyano. Conselleria de Economía Sostenible, Sectores Productivos, Comercio y Trabajo.

Se resumen en este cuadro las fuentes consultadas: 


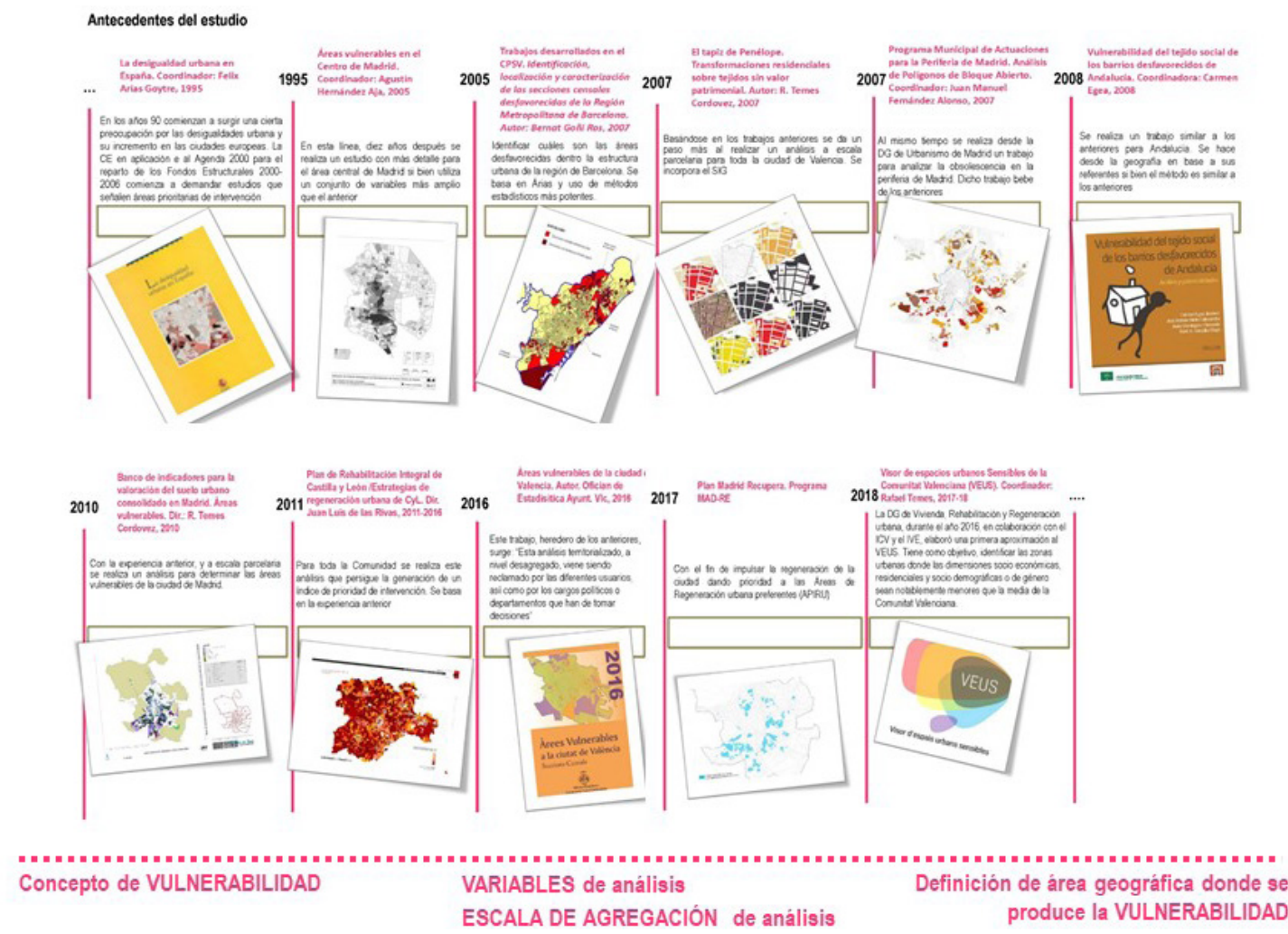

Fig. 1. Resumen cronológico de referencias para el trabajo del VEUS. (Fuente: Elaboración propia)

\section{Tipificación de las variables}

Para poder proceder a sumar variables que están en diferentes escalas y unidades se procede a tipificar las mismas. El primer paso dado en este proceso consistió en la tipificación a partir de cada dimensión a partir de la agregación de las variables. Para ello, los registros de las variables expresados en diferentes unidades de medida $(\% ; \mathrm{e} / \mathrm{m} 2 \ldots)$ se clasificaron en un intervalo de 0 a 3 de manera lineal. El establecimiento de clases o categorías se determina en función del percentil 33 (p33) y del percentil 66 (p66) que representan los valores a partir de los que se encuentran el $33 \%$ de los datos de una variable o el $66 \%$ de los datos de la variable. Con ello se establece la siguiente categorización:

$\begin{array}{ll}\text { Percentil } & \text { Categoría }^{1} \\ 0-\mathrm{p} 33 & \text { BAJA } \\ \text { p33-p66 } & \text { MEDIA } \\ >\text { p66 } & \text { ALTA } \\ & \\ { }^{1} \text { La categoría } & \text { será inversa en función del } \\ \text { sentido de las variable }\end{array}$

Esta clasificación se realizará para cada una de las 3 dimensiones (ResidencialSocioeconómico y Sociodemográfico) obteniéndose una puntuación para cada Sección Censal, según el grado de Vulnerabilidad apreciado.

\section{Factores de ponderación}

Con el propósito de tener en cuenta el diferente grado de vulnerabilidad que es posible detectar entre hombres y mujeres, en determinadas variables se ha introducido un "Factor Corrector" que trata de reconocer esta diferencia. En este Informe se ha utilizado un Factor A (mujeres) de 1,20 (ponderación de un $20 \%$ ) y un Factor B (Hombres) de 1,00 .

En la dimensión "Socioeconómica" tanto en la variable E2 como E3, son de peor calidad que el resto de las variables. El motivo es doble. Por un lado, ya que sus datos no se han conseguido con un nivel de agregación de Sección Censal y por otro, porque la fecha de los datos es de 2011 y 2015. Para matizar esta circunstancia se aplicará un "Factor Ponderador" (Factor 
Tabla 1. Resumen variable de partida agrupadas por campos o áreas. (Fuente: Elaboración propia)

\begin{tabular}{|l|l|l|}
\hline $\begin{array}{l}\text { DATOS } \\
\text { RESIDENCIALES }\end{array}$ & Denominación de dato & Fuente \\
\hline R1 & $\begin{array}{l}\text { SUPERFICIE MEDIA POR HABITANTE DE } \\
\text { LOS INMUEBLES RESIDENCIALES: } \\
\text { Porrentaje calculado dividiendo el total de M2 de la vivienda/ } \\
N^{0} \text { de reridentes }\end{array}$ & $\begin{array}{l}\text { Trabajo previo ICV, 2015. (Datos a nive1 de Sección } \\
\text { Censal) }\end{array}$ \\
\hline R2 & $\begin{array}{l}\left.\text { Valor medio } m^{2} \text { de uso residencial (e/ } m^{2}\right) \\
\text { R3 }\end{array}$ & $\begin{array}{l}\text { Trabajo previo ICV, 2015. (Datos a nivel de Sección } \\
\text { Censal) }\end{array}$ \\
\hline $\begin{array}{l}\text { ACCESIBILIDAD: } \\
\text { Poreentaje calculado dividiendo el total de viviendas principales } \\
\text { accesibles / Total de viviendas principales }\end{array}$ & $\begin{array}{l}\text { Censo 2011. Petición de microdatos confidenciales. } \\
\text { (Datos a nivel de Sección Censal) }\end{array}$ \\
\hline
\end{tabular}

\begin{tabular}{|c|c|c|}
\hline \begin{tabular}{l|l} 
DATOS SOCIO & 1 \\
ECONÓMICOS &
\end{tabular} & Denominación de dato & Fuente \\
\hline E1 & 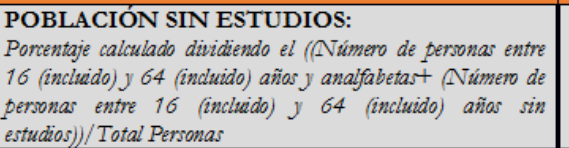 & $\begin{array}{l}\text { Censo 2011. Petición de microdatos confidenciales. } \\
\text { (Datos a nivel de Sección Censal) }\end{array}$ \\
\hline E2 & $\begin{array}{l}\text { TASA DE PARO REGISTRADO ESTIMADO } \\
\text { CON PERSPECTIVA DE GÉNERO } \\
\text { Porrentaje calculado dividiendo (Factor A * Paro Registrado de } \\
\text { Mujeres + Factor B * Paro Registrado de Hombres)/Población } \\
\text { Total entre } 16 \text { y } 64 \text { años }\end{array}$ & $\begin{array}{l}\text { Servicio Público de Empleo Estatal (octubre 2017) y } \\
\text { Padrón Continuo de habitantes, 2016. (Datos a nivel de } \\
\text { Municipal) }\end{array}$ \\
\hline $\begin{array}{l}\text { R } \\
\text { S } \\
\text { I } \\
\text { q } \\
\bullet \\
\text { - } \\
\text { - }\end{array}$ & $\begin{array}{l}\text { RIESGO DE POBREZA Y/O EXCLUSIÓN } \\
\text { SOCIAL: } \\
\text { La población que se encuentra en alguna de las tres situaciones } \\
\text { que se definen a continuacion: } \\
\text { - En riesgo de pobreza ( } 60 \% \text { mediana de los ingresos } \\
\text { por unidad de consumo) } \\
\text { - En carencia material severa (con carencia en al } \\
\text { menos } 4 \text { conceptos de una lista de } 9 \text { ) } \\
\text { - En hogares sin empleo o con baja intensidad en el } \\
\text { empleo (hogares en los que sus miembros en edad de } \\
\text { trabajar lo hicieron menos del } 20 \% \text { del total de su } \\
\text { potencial de trabajo durante el año de referencia) }\end{array}$ & $\begin{array}{l}\text { Portal Estadístico de 1a GV, } 2015 \text { (Datos a nive1 de } \\
\text { Comarca) }\end{array}$ \\
\hline $\begin{array}{l}\text { DATOS SOCIO } \\
\text { DEMOGRÁFICOS }\end{array}$ & \begin{tabular}{|l|l} 
& Denominación de dato \\
\end{tabular} & Fuente \\
\hline D1 & $\begin{array}{l}\text { POBLACIÓN VULNERABLE CON } \\
\text { PERSPECTIVA DE GÉNERO: } \\
\text { Porrentaje calculado dividiendo : (Personas de menos de } 16 \\
\text { años + Factor } A \text { * Mujeres con más de } 64 \text { añost Factor } B \\
\text { * Hombres con más de } 64 \text { años) / Total Personas }\end{array}$ & $\begin{array}{l}\text { Padrón continuo de habitantes, 2016. (Datos a nivel de } \\
\text { Sección Censal) }\end{array}$ \\
\hline D2 & $\begin{array}{l}\text { HOGARES VULNERABLES CON } \\
\text { PERSPECTIVA DE GÉNERO: } \\
\text { Porrentaje calculado dividiendo (Factor } A * \text { Hogar con una } \\
\text { mujer sola de } 65 \text { años o mást Factor B Hogar con un } \\
\text { bombre solo de } 65 \text { años o mást Hogar con padre o madre } \\
\text { que convive con algün bijo menor de } 25 \text { años) / Total } \\
\text { Hogares }\end{array}$ & $\begin{array}{l}\text { Censo 2011. Petición de microdatos confidenciales. } \\
\text { (Datos a nivel de Sección Censal) }\end{array}$ \\
\hline D3 & $\begin{array}{l}\text { POBLACIÓN INMIGRANTE CON } \\
\text { PERSPECTIVA DE GÉNERO: } \\
\text { Porrentaje calculado dividiendo (Factor A } * \text { Mujeres de } \\
\text { nacionalidad extranjera que ban nacido en Europa no } \\
\text { comunitania, Africa, América Central, del sur o Caribe, } \\
\text { Asia) + Factor B * Hombres de nacionalidad extranjera } \\
\text { que ban nacido en Europa no comunitaria, Africa, Aménica } \\
\text { Central, del Sur o Caribe, Aria) / Total Personas }\end{array}$ & $\begin{array}{l}\text { Padrón continuo de habitantes, 2016. (Datos a nivel de } \\
\text { Sección Censal) }\end{array}$ \\
\hline
\end{tabular}

C) que reduzca sus pesos, en la operación de adición final. El factor utilizado reduce el peso de dichas variables un $20 \%$.

Así mismo, los valores de todas las variables en cada sección censal estarán ponderados en función de la población que acogen. Para ello emplearemos el "Factor de Corrección por Población" (FcP). Este FcP se transforma linealmente en el intervalo 1-3. A mayor población la vulnerabilidad aumentará y viceversa. 


\section{Determinación de las tipologías de vulnerabilidad urbana por Sección Censal}

Las 9 variables anteriores son representativas de las 3 Dimensiones o Ejes entorno a los que la disciplina ha concluido puede determinarse el grado de vulnerabilidad en las áreas urbanas. A partir de estas variables agregadas a nivel de Sección Censal para toda la Comunitat Valenciana, se procederá a dar paso a la definición de las distintas "Tipologías de vulnerabilidad urbana" que utilizaremos en este trabajo.

VULNERABILIDAD INTEGRAL

Secciones Censales en las que 3 de las dimensiones de vulnerabilidad están presentes con niveles Altos

\section{POLIVULNERABILIDAD}

El factor común de estas Secciones Censales es que al menos 2 de las dimensiones tienen valores Altos o existen 2 dimensiones Medias y 1 Alta

\section{VULNERABILIDAD RESIDUAL}

Secciones Censales con el resto de combinaciones posibles

En la Tabla 2 se resumen las tipologías de vulnerabilidad citadas y el rango de puntuación necesaria para poder formar parte de cada uno de los tipos:

\begin{tabular}{|c|c|c|c|c|}
\hline $\begin{array}{l}\text { Tipologías de } \\
\text { Vulnerabilidad }\end{array}$ & Nivel & Puntuación & $\begin{array}{c}\mathrm{N}^{\circ} \mathrm{de} \\
\text { Secciones } \\
\text { Censales }\end{array}$ & Población \\
\hline Vulnerabilidad INTEGRAL & $A, A, A$ & $21,95-55,80$ & $256(7,4 \%)$ & $530.240(10,7 \%)$ \\
\hline Polivulnerabilidad & $\begin{array}{l}A, A, M \\
/ A, A, B / A, M, M\end{array}$ & $14,76-39,83$ & $1160(33,4 \%)$ & $1.904 .640(38,4 \%)$ \\
\hline Vulnerabilidad RESIDUAL & $\begin{array}{l}M, M, M / A, B, B / M, M \\
B / A, M, B \quad / M, B, B / \\
B, B, B\end{array}$ & $4,80-23,50$ & $2054(59,2 \%)$ & $2.525 .088(50,9 \%)$ \\
\hline
\end{tabular}
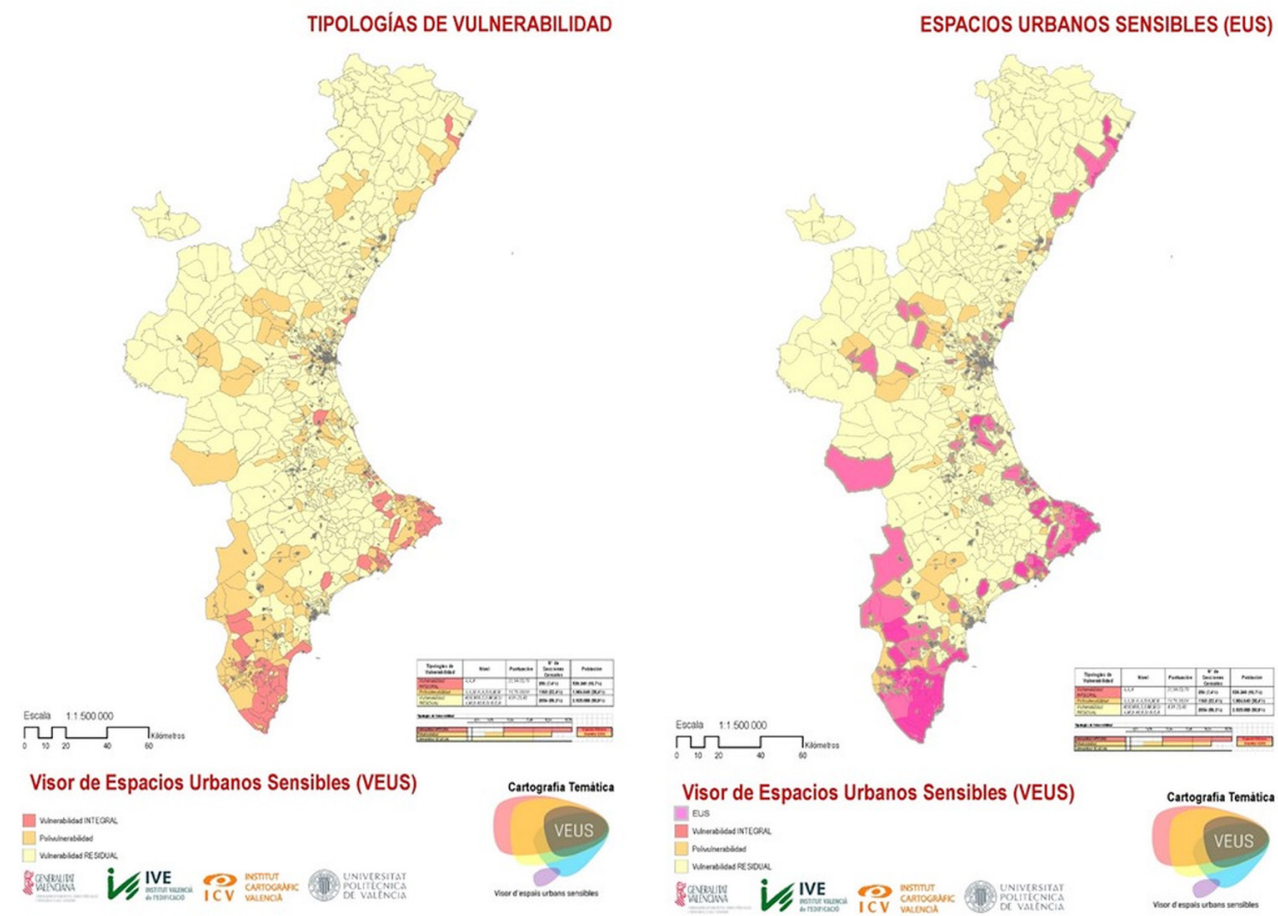

Fig. 2. Tipologías de vulnerabilidad y Espacios urbanos Sensibles de la C. Valenciana. (Fuente: Elaboración propia) 


\section{Delimitación de ámbitos mayores: ESPACIOS URBANOS SENSIBLES (EUS)}

Se definen los "Espacios Urbanos Sensibles (EUS)" como aquellas zonas urbanas delimitadas en base a las distintas dimensiones de vulnerabilidad: vulnerabilidad físicoresidencial; vulnerabilidad socioeconómica; vulnerabilidad sociodemográfica. Para concretar su delimitación se ha optado por definir, como los EUS aquellos en peorsituación: "las agrupaciones de Secciones Censales en las que están presente niveles de Vulnerabilidad INTEGRAL o POLIVULNERABILIDAD” y con una puntuación superior a 21,81 . De esta manera la Figura 3 quedaría:

\begin{tabular}{|l|l|l|l|l|l|}
\hline Tipologías de Vulnerabilidad & \multicolumn{1}{|c|}{ 14,76 } & 21,95 & 23,5 & 39,83 & \\
\cline { 2 - 6 } & & & & & \\
\hline
\end{tabular}

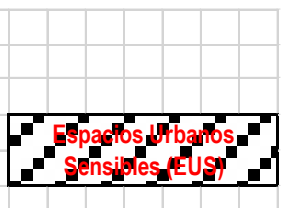

Fig. 3. Tipologías de vulnerabilidad y puntuación. Espacios Urbanos Sensibles de la Comunitat Valencia. (Fuente: Elaboración propia)

De acuerdo con esta definición, los EUS estarían integradas por 830 Secciones Censales en una primera delimitación automática. En esta primera aproximación los EUS representan un $24 \%{ }^{2}$ de las Secciones Censales, y acogen a una población de 1.531.261 personas que representan un $31 \%$ de la población total ${ }^{3}$. (Tabla 3)

Tabla 3. Resumen de espacios Urbanos Sensibles por provincia. (Fuente: Elaboración propia)

\begin{tabular}{|l|r|r|r|}
\hline Provincia & \multicolumn{1}{|c|}{$\mathbf{N}^{\circ}$ de SC } & $\begin{array}{c}\text { N } \text { de } \mathbf{S C} \text { en } \\
\text { EUS }\end{array}$ & $\begin{array}{c}\text { Proporción en } \\
\text { EUS }\end{array}$ \\
\hline Alicante & 1.222 & 458 & $37,47 \%$ \\
\hline Castellón & 458 & 48 & $10,48 \%$ \\
\hline Valencia & 1.808 & 324 & $17,92 \%$ \\
\hline & 3470 & 830 & $\mathbf{2 3 , 9 1 \%}$ \\
\hline
\end{tabular}

\section{Conclusiones}

La existencia de una herramienta que asista a la toma de decisiones y permita priorizar las actuaciones de cara a reducir los problemas de vulnerabilidad urbana integral, resulta de gran utilidad para cualquier administración. $\mathrm{Si}$ la existencia de esta herramienta la administración desarrollaba su actuación de una forma coyuntural, sin tener una idea clara de la magnitud real de los problemas ni la prioridad estratégica de intervención en un ámbito frente a otro. El mapa de vulnerabilidad integral que proporciona el VEUS resuelve en parte esta problemática.
Aun siendo un instrumento de utilidad podemos destacar algunos déficits que progresivamente deberían ir reduciéndose:

-Una limitación es el uso de las secciones censales como unidad espacial de delimitación de los datos. Su estructura es dinámica, por lo que al depender del número de habitantes para su delimitación, la variación por incremento de población o descenso de la misma produce cambios de una recogida de datos a otra (de censo a censo). Así nos encontraremos con secciones que entre dos Censos coinciden perfectamente, secciones que quedan integradas en una nueva delimitación, y secciones que son divididas para crear varias nuevas delimitaciones. Adicionalmente a este déficit, los territorios del interior con menor problamción quedan peor represetnados que los de grandes ciudaes donde el grado de distinción espacial es mucho más preciso.

-Otro aspecto que consideramos debe ser valorado mejor, es la ponderación positiva que se produce al índice o nivel de vulnerabilidad en función del tamaño de población. Establecer una correlación positiva en función de la población, justificando el interés prioritario de la administración en ámbitos donde se afecte a un mayor porcentaje de población. Ahora bien, este principio no necesariamente debe ser interpretado de esta forma. El fenómeno del despoblación que están sufriendo muchos territorios del interior de la Comunitat 
contribuye de forma directa a incrementar la vulnerabilidad de estas zonas por lo que dicho aspecto entra en contradicción con el factor de ponderación utilizado.

\section{Agradecimientos}

Agradezco al Instituto Valenciano de la Vivienda (IVE) y a su gerente Dr. Begaoña Serrano Lanzarote la coordinación del trabajo del VEUS junto con las Directrices para el desarrollo de estrategias de regeneración urbana para municipios de la Comunitat Valenciana. Así mismo agradezco al Instituto Cartográfico Valenciano (ICV) el trabajo realizado para poder alojar el Visor en su plataforma de información.

\section{Notas}

1 La justificación de esta ponderación está respaldada por las conclusiones de varios trabajos entre los que se destacan: Llano Ortiz, J. C. (2015). El estado de la pobreza. Seguimiento del indicador de riesgo de pobreza y exclusión social en España 2009-2013; Valls, F., \& Belzunegui, Á. La pobreza en España desde una perspectiva de género. Fundación FOESSA, 2014.

2 Se trata de un porcentaje muy próximo a la Tasa AROPE (At Risk Of Poverty and/or Exclusion) 2016 de la Comunitat Valenciana, situada en un $30,5 \%$. Llano (2015)

3 Sobre una Población total de 4.959.968 habitantes. Padrón Continuo a 1 de enero de 2016

\section{Referencias}

French-Davis, R. (1999). Macroeconomía, comercio y finanzas para reformar las reformas en América Latina. Santiago de Chile: McGraw-Hill Interamericana.

Llano Ortiz, J. C. 2015. “El estado de la pobreza. Seguimiento del indicador de riesgo de pobreza y exclusión social en España 20092013”. En Valls, F., \& Belzunegui, Á. La pobreza en España desde una perspectiva de género. Fundación FOESSA, 2014

Pizarro, R. 2001. La vulnerabilidad social y sus desafíos: Una mirada desde América Latina.
Serie Estudios Estadísticos y Prospectivos 6. Santiago de Chile: Comisión Económica para América Latina (Cepal), http://www.eclac. org/publicaciones/xml/3/6553/lcl1490e.pdf

Temes, R. R. 2014. "Valoración de la vulnerabilidad integral en las áreas residenciales de Madrid". EURE (Santiago), 40(119), 119-149. doi: 10.4067/S025071612014000100006

Temes, R. 2007. El tapiz de Penélope. Transformaciones residenciales sobre tejidos sin valor patrimonial (Tesis doctoral, Universidad Politécnica de Valencia, Valencia, España). Recuperada de http://hdl. handle.net/10251/2906

Townsend, P., Jarman, B., Jarman, B., Marmot, M. G., Adelstein, A. M., Robinson, N., Thunhurst, C. 1987. "Deprivation". Journal of Social Policy, 16(2), 125. doi: doi. org/10.1017/S0047279400020341

Valls, F., \& Belzunegui, Á. 2014. La pobreza en España desde una perspectiva de género. Fundación FOESSA, 2014

Bustamante, J. 2000. "Un marco de referencia acerca de la vulnerabilidad de los migrantes como sujetos de los derechos humanos". Artículo presentado en Taller Sobre Mejores Prácticas en Materia de Migración, Comisión Económica para América Latina y el Caribe (Cepal), Santiago, Chile.

Rodríguez, J. 2001. Vulnerabilidad y grupos vulnerables: un marco de referenciaconceptual mirando a los jóvenes. Serie Población y Desarrollo 17. Santiago de Chile: Comisión Económica para América Latina (Cepal), http://www.eclac.org/ publicaciones/xml/9/7889/1c11588-P.pdf

Alguacil, J. 2006. "Barrios desfavorecidos: Diagnóstico de la situación española". En F. Vidal Fernández (Ed.), V Informe FUHEM de políticas sociales: La exclusión social y el estado del bienestar en España (pp.155-168). Madrid: FUHEM

Egea, C., Nieto, J. A., Domínguez, J., \& González, R. A. 2008. Vulnerabilidad del tejido social de los barrios desfavorecidos de Andalucia. Sevilla: Centro de Estudios Andaluces, Consejeria de la Presidencia. 